\title{
Active control of locomotion facilitates nonvisual navigation
}

\author{
John W. Philbeck ${ }^{1}$, Roberta L. Klatzky ${ }^{1,2}$, Marlene Behrmann ${ }^{1,2}$, \\ Jack M. Loomis ${ }^{3}$, and Jeremy Goodridge ${ }^{2}$
}

${ }^{1}$ Department of Psychology

Carnegie Mellon University

Pittsburgh, PA 15213

${ }^{2}$ Center for the Neural Basis of Cognition

Pittsburgh, PA 15213

${ }^{3}$ Department of Psychology

University of California, Santa Barbara

Santa Barbara, CA 93106

\section{Running Head: NONVISUAL NAVIGATION}

Keywords: walking, locomotion, triangle completion, dead reckoning, visually directed action, spatial updating, path integration 


\begin{abstract}
In some navigation tasks, participants are more accurate if they view the environment beforehand. To characterize the benefits associated with visual previews, we guided 32 blindfolded participants along simple paths and asked them to walk unassisted to a specified destination (e.g., the origin). Paths were completed without vision, with or without a visual preview of the environment. Previews did not necessarily improve nonvisual navigation. When previewed landmarks stood near the origin or at off-path locations, they provided little benefit; by contrast, when they specified intermediate destinations (thereby increasing the degree of active control), performance was greatly enhanced. The results suggest that the benefit of a visual preview stems from the information it supplies for actively-controlled locomotion. Accuracy in reaching the final destination, however, is strongly contingent upon the destination's location during the preview.
\end{abstract}

\title{
Active control of locomotion facilitates nonvisual navigation
}

For many people, navigating to locations in the nearby environment is a straightforward task. Vision typically affords many sources of information that are useful for keeping track of one's changing position and orientation when walking. It is not uncommon, however, to encounter situations in which navigation must be accomplished without vision - for example, when attempting to maneuver in an environment that has suddenly become dark or when walking from a brightly lit area into a deeply shadowed one. In these situations, one must sense the progress of one's movement by monitoring internally-generated self-motion signals, such as changes in the position of the limbs and muscles or acceleration signals from the vestibular organs of the inner ear. By integrating signals specifying one's own velocity, or doubly integrating acceleration signals, one may arrive at an estimate of one's current position and orientation. This method of self-motion updating is called path integration or dead reckoning (Gallistel, 1990; Loomis, Klatzky, Golledge, \& Philbeck, 1999); it excludes forms of updating which rely upon acquiring positional information by directly sensing features of the environment (e.g., seeing a landmark at a known location).

By this definition, path integration does not exclude all forms of information obtained by directly sensing the environment--only those that supply information about the navigator's position. Thus, vision can provide an input to path integration via optic flow when the flow conveys information about the navigator's velocity. Honeybees, for example, have been shown to rely heavily upon this form of information when navigating (Srinivasan, Zhang, \& Bidwell, 1997). A very distant environmental feature that is directly sensed through vision or audition could also enhance path integration by providing an azimuthal reference, even though this reference does not supply the information necessary for position fixing.

Our interest here, however, is in the processes underlying path integration when direct sensing of environmental features is precluded, and only information about the navigator's motion through the environment is available. Although some studies suggest that humans are quite good at performing path integration under these challenging conditions, others have concluded that humans make sizable systematic errors. Our aim in this paper is to consider some of the factors that may be relevant for facilitating or inhibiting path integration performance and to test among some of these possibilities.

Perhaps the most direct way of assessing the ability to sense changes in location due to walking is to guide blindfolded participants along a straight path, and then ask them to estimate how far they have walked, either verbally or by attempting to reproduce that distance (Klatzky et al., 1990; Loomis et al., 1993; Philbeck, Behrmann, Black, \& Ebert, in press). Although performance is not markedly inaccurate, there is a tendency to overestimate short walked distances 
(within $4 \mathrm{~m}$ ) and underestimate longer ones. When locomotor turns are reproduced while blindfolded they tend to be underestimated (Klatzky et al., 1990). Another way of testing path integration ability, using slightly more complex paths, involves guiding participants along two straight path segments separated by a turn, and then asking them to indicate the starting point of the path by pointing to it (Sadalla \& Montello, 1989). Again, participants tend to make systematic errors; these errors are modulated by the magnitude of the turn separating the two straight path segments. Accuracy drops even more when additional turns and straight path segments are included (Klatzky et al., 1990).

One multiple-segment test of path integration is the "triangle completion" task (Loomis et al., 1993; Sholl, 1989; see also Berthoz et al., 1999). Triangle completion studies are typically conducted with only a limited amount of visual exposure to the walking environment, if any. Participants are blindfolded and guided along two straight path segments, separated by a turn. At the end of the second straight segment, they are released and attempt to locomote back to the starting point unassisted. The response trajectories, however, generally do not converge, and the centroid of the terminal points is displaced from the origin (Loomis et al., 1999). Participants tend to overshoot the correct response turns and path lengths when relatively small values are required and undershoot when relatively large response values are required (Klatzky, Beall, Loomis, Golledge, \& Philbeck, 1999; Loomis et al., 1993). Although this might appear to be a range or context effect, such as is found in certain other motor behaviors (e.g., Pepper \& Herman, 1970), pure range effects do not appear to account for the errors in triangle completion tasks. For example, Klatzky et al. (1999, Experiment 1) found that manipulation of the response range across blocks of trials had little effect on errors, and the point at which the direction of error reverses (from overshoot to undershoot) in these tasks is not reliably close to the mean of the response range. Accordingly, the pattern of inaccuracy in triangle completion has been interpreted as reflecting systematic errors in path integration, rather than as a range effect (Loomis et al., 1999).

Taken together, the foregoing studies paint a poor picture of human path integration ability. Other lines of research, however, suggest that under some circumstances humans are actually quite good at sensing their self-motion and using it for navigation. In these studies, vision of the environment is supplied before movement begins, but after this visual preview, position updating again must be accomplished by monitoring internally-generated self-motion signals. After first previewing a target in a well-lit environment, blindfolded participants are remarkably accurate and precise when attempting to walk directly to the target, even when it is initially up to $20 \mathrm{~m}$ away (Corlett, 1992; Elliott, 1987; Loomis, Da Silva, Fujita, \& Fukusima, 1992; Philbeck \& Loomis, 1997; Rieser, Ashmead, Talor, \& Youngquist, 1990; Steenhuis \& Goodale, 1988; Thomson, 1983). Likewise, participants accurately point to or turn to face a previously viewed target after first walking without vision to a new location (Fukusima, Loomis, \& Da Silva, 1997; Loomis et al., 1992).

Philbeck, Loomis, and Beall (1997) studied a similar task, involving "indirect walking" (see also Loomis, Klatzky, Philbeck, \& Golledge, 1998). In this task, the participant viewed a destination marker, then covered the eyes and walked along a path oblique to the destination until a tactual marker was reached. At that point the task was to make the appropriate turn and walk the rest of the way to the destination without assistance. When participants previewed the target under well-lit viewing conditions before beginning to walk without vision, the response trajectories not only converged, but also clustered around the physical target location. This suggests that path integration was performed well. These results stand in sharp contrast to the poor performance typical in triangle completion studies, in which the walking trajectories neither converge nor cluster around the physical location of the destination.

The sensory information available to the participant for path integration is virtually the same in triangle completion and indirect walking tasks. What, then, can account for the large 
performance differences? A very general explanation is that information obtained during visual previews of the local environment can powerfully facilitate the sensory processes underlying path integration, even though vision of the environment is not available during the walk. Some animal species (e.g., hamsters) do not appear to benefit greatly from the experience of seeing environmental landmarks before beginning nonvisual locomotion (Etienne et al., 1998); visual previews may play a very different, and more prominent, role in human navigation. Our aim here was to gain a clearer understanding of the processes by which visual previews of the environment facilitate human path integration when vision is no longer available.

\section{Triangle Completion Contrasted with Indirect Walking}

We compared two behavioral paradigms described above which produce striking differences in performance depending on whether or not a visual preview of the environment is supplied: indirect walking and triangle completion by walking. Both are goal-directed tasks, and the performance differences between them can be summarized as (a) convergence of walking trajectories, with clustering near the physical goal location, in indirect walking and (b) failure to converge, and lack of clustering near the goal, in triangle completion.

One obvious difference between the two tasks is in the complexity of their paths: counting both the outbound and response paths, there are three legs and two turns in triangle completion, and only two legs and one turn in the indirect walking tasks studied to date (Loomis et al., 1999). There is evidence that increasing the number of legs and turns in the outbound path tends to degrade performance (Klatzky et al., 1990; Sholl, 1989). However, participants make systematic pointing errors even on tasks involving two legs and one turn on the outbound path (Sadalla \& Montello, 1989), so path complexity by itself is not likely to provide a complete explanation of the performance differences in triangle completion and indirect walking. In the experiment we report below, we controlled for path complexity by restricting the parameters of the paths tested to three legs and two turns.

Assuming that increased path complexity is not the main explanation for the relatively poor performance in triangle completion tasks, we explored other alternatives that focus on aspects of the visual information that might facilitate path integration. Each of the section headings below identifies a potentially relevant difference between indirect walking tasks (with a visual preview) and triangle completion tasks (without a visual preview). These differences are discussed and formulated as hypotheses about the mechanisms by which path integration might be facilitated. The hypotheses ultimately form the basis for manipulations in the experiment presented below.

\section{(1) Remembered Context vs. "Virtual Ganzfeld"}

A visual preview may facilitate path integration by promoting the generation of a structured memory of the environmental layout, thereby expediting the organization of incoming self-motion information. Even if participants begin a triangle completion experiment with a memory of their surrounding environment, it is likely that over the course of several trials without further visual input they become disoriented. Rieser (1999) has argued that a disoriented blindfolded participant experiences a "virtual ganzfeld", a featureless, unstructured impression of the environment. Participants thus deprived of a well-structured frame of reference could be at a disadvantage when performing path integration. Rieser and Frymire (1995; see also Rieser, 1999) tested this idea in an experiment in which blindfolded participants were guided along simple paths and then attempted to aim a pointer back to the origin of locomotion. Before beginning to walk without vision, one group of participants was instructed to imagine being in a familiar setting; another group was allowed to view the real enviroment before vision was occluded. Presumably, both of these groups had access to the memory of a structured environment when attempting to path integrate. These two groups responded more accurately than participants in the "virtual ganzfeld" group, who 
were denied a strong impression of the local environmental structure by being disoriented before exposure to the stimulus paths.

\section{(2) Remembered Landmark vs. No Landmarks}

In addition to the benefit potentially provided by a remembered environmental structure, there may also be a benefit associated with specific remembered features of the environment. Particularly salient locations could be selected as "landmarks". After vision has been obscured, these landmarks are no longer directly sensed and thus cannot provide any information about the participant's position or orientation. Nevertheless, they could serve important functions as reference points for organizing incoming information, as spatial place holders to ameliorate demands on working memory, or as mobilizers of attention. Remembering very distant objects (e.g., the sun) could facilitate path integration by providing an azimuthal reference; remembering nearby objects could provide a distinct source of facilitation by making use of knowledge of the parallax that nearby objects undergo during locomotion. Rieser, Frymyre and Berry (1997) found that performance on a multi-segment path integration task improved after participants viewed a configuration of nearby landmarks versus a configuration of directionally equivalent distant landmarks, even though in both conditions participants walked and responded without vision. Presumably, updating was facilitated by knowledge that under sighted conditions, the nearer targets would produce greater optical flow as the participant walked. Interestingly, the highly expert maritime navigators in the Republic of Micronesia, who successfully sail great distances without physical maps or instruments, cite the use of very distant imagined landmarks ("etaks") as a powerful aid in maintaining accurate position and orientation estimates while out of sight of land (Gladwin, 1990; Hutchins, 1995).

\section{(3) Visual Specification of Target vs. Non-Visual Specification of Target}

Visual previews also enhance the perceptual localization of the destination. When triangle completion is conducted without any vision of the environment, the sensory information specifying the goal location differs greatly from that available in indirect walking. In triangle completion, the origin/goal is typically specified in an abstract, non-visual manner (e.g., by a cessation in walking of several seconds before the next trial begins), whereas in indirect walking, the goal is specified visually. There is evidence that the availability and fidelity of information specifying a destination's location systematically influences goal-directed walking (Philbeck et al., 1997). It may be that the relatively poor path integration performance seen in typical triangle completion tasks reflects the relative ineffectiveness of non-visual means of specifying the goal location.

\section{(4) Active Control vs. Passive Control}

Another factor that could be relevant for path integration ability is how much of the path is specified by the experimenter and how much is generated under the participant's control. In indirect walking, the participant follows an experimenter-defined path along one straight segment, and then produces one turn and one straight path segment without experimenter guidance. In triangle completion, the participant follows along two experimenter-defined straight path segments as well as an experimenter-defined turn. Indirect walking performance could be enhanced because it provides a higher proportion of participant-guided path segments.

Movements that are actively controlled by an individual potentially provide several sources of information that might facilitate path integration, over and above those provided by passive movements (motion not directly controlled by the individual). Both active and passive movements produce changes in muscle lengths and joint angles that can potentially provide feedback ("inflow") information about self-motion. Active movements, however, are also associated with feedforward ("outflow") signals serving to actuate movement of the muscles, and might therefore have more to contribute to self-motion sensing. The notion that outflow signals play a distinct role in updating eye movements, for example, has enjoyed a long history (Festinger, Ono, \& Burnham, 1967; Helmholtz, 1962). 
More generally, the distinction between active and passive control can apply at more abstract, hierarchical levels. In path integration tasks, walking is undeniably "active" during production of the response path, but even when walking is to some extent controlled by the experimenter, it is not "passive" in the sense that only feedback information about movement is generated. On the contrary, the brain must still send feedforward commands to actuate the leg muscles, just as it does during active production of the response path. In such tasks, the active/passive distinction implies another source of information, in addition to "outflow", that could potentially facilitate path integration. This information is related to knowledge about an upcoming movement. When performing a triangle completion task, a participant undergoing an experimenter-defined turn does not know what turn magnitude the experimenter will select. By contrast, a participant who has seen an intermediate destination has information upon which to base an estimate of the turn that will be required. For example, the participant could generate a movement plan from the initial viewpoint, and then modify it during the experimenter-defined path segment according to incoming self-motion information. Alternatively, the participant could simply update the target's location during the experimenter-defined segment and then generate a movement plan just before executing the response turn. Participant-defined and experimenterdefined movements have been termed "preselected" and "constrained", respectively, to capture this distinction in prior knowledge about an upcoming movement. "Preselected" arm movements are typically reproduced with greater accuracy and precision than "constrained" movements (Kelso, 1977; Summers, Levey, \& Wrigley, 1981), and similar underlying processes could act to faciliate path integration when the walking trajectory entails more "active" segments.

\section{(5) Origin Destination vs. Non-Origin Destination}

Finally, it may be that the perception and action systems have become optimized for updating relative to locations that are displaced from the origin (Loomis et al., 1999). Although animals must sometimes update the progress of their motion relative to some origin of locomotion (e.g., relative to a nest during foraging behavior), locomotion is perhaps more frequently directed toward other destinations. In humans and many other species, forward locomotion is predominant, and as such walking is usually directed to destinations in front of the individual. Although the origin itself could also serve as a forward destination, in many cases the goal of returning to the origin is subordinated to more immediate ones that are associated with other forward destinations. Over time, non-origin destinations (particularly those lying in front) may have come to play a privileged role in the performance of path integration. In the context of triangle completion studies, one consequence of having the origin as a destination is that it immediately passes behind the participant as soon as he or she begins to walk along the first path segment. This might disadvantage updating in triangle completion tasks.

\section{Experiment}

Our aim here was to evaluate the individual contribution of a number of the factors discussed above, in hopes of uncovering which are most relevant for facilitating human path integration. To do this, we tested performance on several variations of the methods used in typical triangle completion experiments. Four groups of participants participated in two experimental blocks. In Block 1, we established a baseline level of performance on the standard triangle completion task by asking all participants to attempt to return to the origin of locomotion after being guided by an experimenter along two straight path segments and the intervening turn. The participants were not permitted vision of the workspace during these trials. In Block 2, the participants viewed the workspace before each trial, and then covered their eyes before walking began. With regard to Hypothesis (1) above (Facilitation by Remembered Context), we would expect each group to show improvement across blocks if having access to a memory of the surrounding environment improves path integration. 
Insert Figure 1 about here

The groups were distinguished in Block 2 by the specific task performed and the visual information available during the preview (see Figure 1). In the "OFF-PATH" group, we tested the effect of seeing a salient marker in the workspace which could be used as a nearby remembered landmark (Hypothesis 2). This marker was never positioned directly on the walking path, but was instead placed at a location randomly selected out of 4 possibilities. In the "ORIGIN" group, we tested the effect of seeing the destination (Hypothesis 3) by placing a marker very near the origin. Although the origin was visible to all groups in Block 2 during the visual preview, we thought that placing a marker there would provide particularly strong visual information about the origin / goal location. Note that although this marker lay on the participant's path (at the ideal endpoint of the path), it did not provide the participant with prior knowledge of the magnitude of any turns or straight segments that should occur in the response trajectory. In the "ON-PATH" group, we increased the proportion of actively controlled path segments to test Hypothesis (4). We did this by placing a marker at an intermediate destination on the path. This condition takes the indirect walking task in Philbeck et al. (1997) and adds the requirement of returning to the origin after first walking to the marker: after the visual preview, the participant was guided without vision along a single straight path segment. At the end of this segment, the participant attempted to walk to the previously viewed marker, and then to turn and walk the rest of the way back to the origin. In this way, at the end of the first path segment, the participant could use the known marker location to compute the intended magnitude of the first turn and intended length of the second path segment before executing them. This knowledge was not present for the OFF-PATH and ORIGIN groups. ON-PATH participants could also use the visual preview to encode in advance the length of the third leg, which lay between the origin and the intermediate marker, but the earliest point at which they could determine the required response turn was when they were stopped at the end of the first leg.

Finally, in the "ROTATED" group, we tested the notion that path integration may be improved when it is performed with respect to a destination that is displaced from the origin during the preview (Hypothesis 5). This group did not perform triangle completion per se in Block 2, but instead attempted to walk to two locations in sequence, neither of which was the origin. The participants began by seeing two markers during the visual preview. The first, as in the ONPATH group, was placed at an intermediate destination on the path; the second marked a destination that was not coincident with the origin (see Figure 1a). The destination points were mirror images of the location of the origin, reflected about the second path segment. A participant performing perfectly would walk along the same first two path segments as the participants in all other groups. However, instead of making a right turn to walk back to the origin, participants in the ROTATED group would make a left turn of the same magnitude, and then walk the same distance to reach the final destination. Thus, at the end of the first path segment, the ON-PATH and ROTATED groups were both able to compute the intended first turn and second path segment (Hypothesis 4) but only the ROTATED attempted to walk to a non-origin destination (Hypothesis 5). Like the on-path group, the rotated group could also compute the length of the third leg in advance of beginning the path, but could not pre-compute the response turn.

We predicted that all groups would show some improvement between blocks, due to the availability in memory of the structure of the surrounding environment (Hypothesis 1). We expected that conditions in which the participant actively controlled a larger proportion of the walking trajectory would yield the best performance (supporting Hypothesis 4). Thus, we thought that both the ON-PATH and ROTATED groups would perform especially well in Block 2 because their visual preview of an intermediate destination would allow them to generate intended trajectory elements before actually executing them. In spite of the reasons to expect walking to a non-origin goal to be performed more accurately than walking back to the origin (Hypothesis 5), our intuition 
was that a non-origin destination would be less well-localized than the origin itself, and that this might degrade performance of the ROTATED group relative to the ON-PATH group. We predicted that the results of the other groups would confirm that the relatively good performance of the ON-PATH and ROTATED groups was not due to seeing a salient landmark (Hypothesis 2) or to visual specification of the destination (Hypothesis 3). We thought, however, that seeing a marker at the origin might provide a slight advantage by way of increasing the salience of that location. Thus, from best to worst, the predicted pattern of performance after visual previews in Block 2 was: ON-PATH, ROTATED, ORIGIN, OFF-PATH.

\section{Participants}

\section{Method}

Thirty-two individuals from the Carnegie Mellon community consented to participate in this study and were paid $\$ 7$ each. The average age was 21 (range 17 - 49). The participants were randomly assigned to participate in one of 4 treatment groups, with 8 participants in each group (4 male, 4 female). Data from 13 others were collected but excluded from analysis; this screening was necessary to equate performance between groups in the first block. We decided to exclude data from observers whose mean response turn errors in the first block exceeded 40 degrees. Although individual mean response turn errors exceeding this cut-off were not outliers in the statistical sense (roughly $30 \%$ of the participants produced large turn errors), they were each more than 2 standard deviations above the mean of the points falling below it. Nine participants were excluded on this basis. The data from 4 other participants were excluded because a relatively large proportion (over 7\%) of walking trajectories passed near the laboratory walls, necessitating a premature termination of the path for safety reasons. Premature path termination provides error feedback, a source of information we wanted to minimize in our experimental population. Large turn errors and/or substantial veering were conspicuous antecedents of these terminated trajectories. Response path length errors were more evenly distributed and no cut-off was necessary.

\section{Design and Stimuli}

Four groups of participants took part in each of two experimental blocks. In Block 1, all participants performed under identical conditions, attempting to complete 30 triangles without vision of the workspace to establish a baseline level of performance; in Block 2, all participants viewed the workspace before each trial, but the 4 groups were distinguished by the task performed and the visual information available during the preview of the workspace. The task and visual information manipulations will be described in more detail below. The stimulus paths consisted of 12 triangles (see Figures $1 \mathrm{~b}$ and $1 \mathrm{c}$ ). We adopt the following naming convention for the legs and turns of the stimulus and response paths: the first two straight paths will be called Legs A and B, and the intervening first turn will be called Turn 1. At the end of Leg B, the participant actively produced Turn 2 (the response turn) and Leg C (the response path length) when attempting to walk to the final destination. Six experimental paths were measured 3 times apiece in each block. These triangles all shared the same Leg A length of $3 \mathrm{~m}$, followed by a Turn $1 \mathrm{of} 40,75$, or $110 \mathrm{deg}$, and then a Leg B of 2.5 or $5 \mathrm{~m}$. Six other paths were measured twice apiece per block, and consisted of a Leg A of 2, 4, or $5 \mathrm{~m}$, a Turn 1 of $130 \mathrm{deg}$, and then a Leg B of 1.5 or $3.5 \mathrm{~m}$. These were initially intended to be "dummy" paths, which would extend the range of ideal response turns and response path lengths, and encourage participants to process each leg of the outbound path rather than assuming that the first leg was always the same. However, data were ultimately analyzed for all paths, as the findings were the same for the total set of paths as for those tested three times per block. The presentation order was fully randomized within each block.

\section{Apparatus}

The experiment was conducted in two large indoor gymnasia (31 x $13 \mathrm{~m}$ and $21 \times 13 \mathrm{~m})$. Each subject participated in only one gymnasium. Both workspaces were uncarpeted, well-lit, and free from support columns and clutter. Although the gymnasia differed somewhat in terms of wall treatments (e.g., window placement), these features were fairly distant from the participant's 
starting position. The position of the far wall with respect to the walking trajectories was the same in both spaces--participants began each trial $10 \mathrm{~m}$ away from the far wall and directly facing it. Numerous pieces of tape were visible on the floor of both spaces, but only some of these were relevant for this study as markers for stimulus locations and/or turning points for the stimulus paths. Due to the limited availability of these large spaces, we could not completely counterbalance the number of participants across workspace and group. The number of participants in each group run in the smaller gymnasium was 6, 5, 6 and 3 for the ORIGIN, OFFPATH, ON-PATH and ROTATED groups, respectively; the rest were run in the larger space. ${ }^{1}$

To minimize environmental sounds that could provide auditory cues to the participant's location and orientation in the workspace, the participants wore foam earplugs inserted into the external auditory canal and hearing attenuators covering the entire external ear. Participants wore a blindfold when walking in the experiment. When guided along a path by the experimenter, the participants grasped a bar $(35 \mathrm{~cm})$ with both hands. The experimenter grasped the bar in its center with one hand and guided the participant by walking along in front of him or her. Upright music stands (about $120 \mathrm{~cm}$ tall) were used as location markers in Block 2. The stands had a round base and an opaque, one-piece top that was oriented to face the participant.

\section{Procedure}

Block 1. The participants were told that they would be guided along the first two legs of a triangular path and the intervening turn. The instructions specified that at the end of the second leg, the participants were to attempt to complete the triangle by returning to the origin of locomotion unassisted, following the most direct path. Before entering the workspace for the first time, the participants donned the hearing attenuation devices and the blindfold; thereafter, they began each trial oriented in line with the first straight path segment. The experimenter signaled the initiation of each trial by raising and lowering the bar. The experimenter guided each participant along the first leg of one of the 12 paths, turned through an angle, and then continued on along the second leg. At that point, the participant turned and attempted to walk directly back to the origin without assistance. The stopping location was determined via trilateration by taking a tape measurement from that location to each of two known points. After the response, the experimenter led the participant along a winding path back to the true origin. No error feedback was given.

Block 2. Participants in all groups except for the ROTATED group were instructed that the same stimulus paths would be used as in Block 1. Before each trial, all participants raised the blindfold for several seconds to view the workspace. They were oriented in line with the first straight path segment, although none of the stimulus paths was explicitly visible during the visual preview. Depending on the group, participants saw either one or two music stands in the workspace serving as location markers. An assistant either stood next to the single marker or next to the marker at the intermediate destination (end of Leg B) in the ROTATED group. The markers were removed during the short interval between donning of the blindfold and onset of walking.

Group 1 (OFF-PATH) participants saw a single marker at a location randomly selected from one of four possibilities (see Figure 1d). Two of these were to the left of straight ahead from the participant's initial viewpoint, and thus were relatively far from any point on the outbound paths; the other two were on the right of the participant's viewpoint, and thus were closer to the set of stimulus trajectories. Although the marker's proximity to the walking path may systematically influence path integration, we wanted to test for a more general facilitatory effect of off-path markers across a range of proximities. Participants in this group were instructed that paying attention to the remembered location of the marker as they walked might help them to perform the task. After viewing the marker, the participants lowered the blindfold and the trials were conducted without vision as in Block 1. 
Group 2 (ORIGIN). During the visual preview, participants in this group saw a single location marker about $30 \mathrm{~cm}$ in front of them but below eye height so that it did not obscure vision of the workspace. Group 2 participants received similar instructions as Group 1 concerning the potential benefit of the marker. After several seconds, participants lowered the blindfold, and the rest of the trial was conducted as in Block 1.

Group 3 (ON-PATH). During the preview, participants saw a single marker placed at the end of the second segment of one of the same paths used in Block 1. The task was again triangle completion, but instead of being guided to the end of Leg B by the experimenter, the participants were guided to the end of Leg A and released. At that point, they attempted to walk without assistance to the marker's location and then back to the origin, all without vision. Performance in reaching both locations was measured via trilateration, as described above, but the participants received no feedback about the accuracy in reaching either point.

Group 4 (ROTATED) participants saw two location markers during the visual preview, each identified by an easily visible numeral "1" or "2" (see Figure 1e). As in the ON-PATH group, the participants were guided to the end of Leg A and then released. At that point, they attempted to walk without experimenter guidance to marker " 1 " and then to marker " 2 ". Marker "1" was located at the end of Leg B; marker " 2 " was positioned at a location that was a mirror image of the origin's location, reflected about Leg $\mathrm{B}^{2}$. The participants were instructed to turn in the direction that resulted in the shallowest turn to get to the target location. This meant that correct performance entailed first making a clockwise turn to get to marker "1", then a counter-clockwise turn to get to marker "2". Performance in reaching both markers was again measured via trilateration.

\section{Results}

A small number of trials (about 1\%) were omitted due to measurement errors or premature path termination for safety reasons. Using the coordinates of the remaining stopping points, we derived two primary response measures: the response turn, computed relative to the participant's heading at the end of the preceding leg, and the response path length. For the ON-PATH and ROTATED groups, we also derived response turns and path lengths for Turn 1 and Leg B, which were participant-controlled. Because the ROTATED group in Block 2 made a counterclockwise Turn 2 instead of clockwise as the other groups did, we converted each of their Turn 2 values to its equivalent reflected about Leg B.

Unlike previous triangle completion studies involving walking (Klatzky et al., 1999; Loomis et al., 1993), our participants in Block 1 (No Visual Preview) showed very little tendency on average to overshoot or undershoot the ideal response path length or turn. This lack of systematic error was probably due to the fact that we intentionally screened out participants who produced large turn errors. The average absolute turn and path length errors in our sample were 18 degrees and $.83 \mathrm{~m}$, respectively. These errors produced an average straight-line distance error from the final destination of $1.9 \mathrm{~m}$. Thus, the errors that existed before the visual context manipulations, though perhaps smaller than those of the population at large, did allow sufficient room for improvement to test our hypotheses.

We first verified that the four groups did not differ in their baseline performance before the manipulations in Block 2. Analyses of variance (ANOVA) on the Turn 2 and Leg C responses in Block 1 showed there were no reliable differences between groups $(\mathrm{F}(3,28)=.41$ and .66 , MSe $=$ 1278 and 3.8, respectively; both p's > .05). We included Block as a factor in subsequent analyses because changes relative to the Block 1 baseline were of interest.

Insert Figure 2 about here 
Figure 2 shows the centroids of the stopping points for each group, both with and without a visual preview. Only the participants in the ON-PATH group reached the final destination accurately in Block 2; although they made errors in reaching the intermediate destination, their final trajectories nevertheless brought them back in close proximity to the origin. The ROTATED group made similar errors in reaching the intermediate destination, but because their final trajectories failed to compensate for those errors, performance in reaching the final destination was poor. The ORIGIN and OFF-PATH groups, who were guided to the intermediate destination (hence without error), showed almost no change in performance across blocks, despite seeing the workspace before every trial in Block 2.

In Block 2, the ON-PATH and ROTATED groups actively walked to the intermediate destination and generally made errors when doing so. This means that the data for these groups in Block 2 were influenced by an additional source of systematic and variable error that was not present in the rest of the data. Thus, the raw data, and error scores derived from them, are not strictly comparable across both blocks for all groups. We therefore turned to an analysis based on summary measures of performance to focus on group differences in systematic error and minimize differences in variable error. First, we note that the relation between the required response parameters (Turn 2 or Leg C) and the values produced by the participant when attempting to return to the origin is typically well-fit by a straight line (Fujita, Klatzky, Loomis \& Golledge, 1993; Klatzky et al., 1999). The slope and intercept of the best-fitting line through each participant's data thus provides a concise characterization of overall performance. Correct performance would entail a slope of 1.0 and an intercept of zero. Using a least-squares criterion, we fit a straight line through each participant's response path and turn data, obtaining a separate fit for the data in each experimental block. The fits were generally good, yielding squared correlations ranging from .21 to .94 with a mean of .68. In fitting the data for the ON-PATH and ROTATED groups in Block 2, we used the participant's actual stopping point at the end of Leg B, rather than the nominal (ideal) stopping point, as the basis for computing the required and response values of Turn 2 and Leg C. Figure 3 shows the average slopes and intercepts across participants. We performed ANOVAs on the data underlying the means shown in Figure 3, with Block included as a within-subject variable. This yielded 4 separate analyses, the details of which are presented in Table 1.

All of these analyses except the Leg $\mathrm{C}$ intercepts showed a main effect of Block, with a tendency toward increasing accuracy in Block 2 (i.e., increasing slopes and decreasing intercepts); except for the Turn 2 intercepts analysis, however, all of these block effects were qualified by a Block $x$ Group interaction. This interaction is especially important with regard to our hypotheses because it compares the degree to which the different groups were affected by the visual preview manipulations in Block 2. Tukey post-hoc analyses showed that for each of the four ANOVAs, the ON-PATH group always reliably increased in accuracy in Block 2 (slopes moving toward 1.0 and intercepts toward zero; 's $<.05$ ), with none of the other groups showing any reliable difference between blocks. The one exception to this was for Leg C intercepts, in which the ROTATED group's intercepts reliably increased in Block 2 (i.e., became less accurate, $\mathrm{p}<.05$ ). These post-hoc analyses suggest that for the most part the main effects of Block were dominated by the increasing accuracy of the ON-PATH group and the decreasing accuracy of the ROTATED group in Block 2. The slight improvements in accuracy in the OFF-PATH and ORIGIN groups could indicate small effects of the stimulus markers or cumulative effects of increasing experience with the task from one block to the next.

Insert Figure 3 about here

Insert Table 1 about here 
Perhaps the most salient feature of Figure 2 is the strong convergence of walking trajectories for the ON-PATH group in Block 2 and the lack of convergence for the OFF-PATH and ORIGIN groups. Although the centroids of the terminal points for the ROTATED group do not cluster near the final target, the trajectories do in fact converge. For this group, there were six destinations instead of only one, with two different trajectories taken to each destination; averaged across paths, the separation between the two centroids for each destination was just $40 \mathrm{~cm}$ (SD across the six paths: $16 \mathrm{~cm}$ ). The mean separation for the analogous paths in the ON-PATH group was $41 \mathrm{~cm}$ (SD: $16 \mathrm{~cm})$, while the mean ORIGIN and OFF-PATH separations were larger and more variable (68 and $88 \mathrm{~cm}$, respectively; SD: 36 and 37). The dispersion of individual participant centroids around the group centroids for each path was somewhat larger for the ROTATED group than for the ON-PATH group, averaging 161 and $90 \mathrm{~cm}$, respectively. The average dispersion of the OFF-PATH and ORIGIN centroids was 106 and $99 \mathrm{~cm}$, respectively. One must bear in mind, however, that these numbers for the "active" groups cannot be directly compared to those of the other two groups. An additional error source exists for the "active" groups because participants controlled their own path to the end of Leg B rather than being reliably guided to it by the experimenter. In summary, there were group differences with respect to variable (unsystematic) error computed between subjects, as measured by dispersion around the centroid. However, there is also the measure of average convergence, in terms of the separation between response centroids for pairs of paths with a common endpoint in the rotated group (i.e., using the analogous pairs in the other groups). This measure shows that on average, the two groups that actively controlled a larger portion of their trajectories tended to cluster on a given target location more tightly than those of the other two groups. The ON-PATH trajectories converged upon the destination, whereas the ROTATED trajectories converged upon locations displaced from the destinations.

\section{ON-PATH vs. ROTATED}

The indirect walking task used in Philbeck et al. (1997) is embedded in the Block 2 tasks for both the ON-PATH and ROTATED groups: as in the previous study, both groups here saw a target, covered the eyes and followed along a path that passed obliquely to the target; at the end of the first path, they were released and attempted to walk to that target unassisted. Thus, we can compare the accuracy in arriving at the intermediate target (at the end of Leg B) with the earlier findings. In contrast to the results of Philbeck et al. (1997), in which the participants' paths tended to converge upon the target location, in the current experiment there was a tendency in both the ON-PATH and ROTATED groups to overwalk and underturn when attempting to walk to the first target. The mean Leg B slopes (i.e., of functions relating observed to correct values) for the ONPATH and ROTATED groups were 1.08 and 0.96 , respectively, with mean intercepts of 0.45 and $0.51 \mathrm{~m}$. The parameters for the ROTATED group indicate that there was a general tendency to overwalk on Leg B throughout the range of correct values, but small values of Leg B were overwalked more than larger ones. The mean Turn 1 slopes were 0.80 for both groups, with intercepts of 11.3 and $3.5 \mathrm{deg}$, respectively. This produced an average straight-line distance error from the actual location of the first target of about $1.3 \mathrm{~m}$. This error is somewhat greater than the $88 \mathrm{~cm}$ mean error found by Philbeck et al. (1997) when their comparable group walked along paths of similar lengths. The greater error in the current study perhaps reflects the influence of the additional task of walking to another destination. There was a trend toward the ON-PATH group walking farther and turning more (i.e., more overwalking and less underturning) than the ROTATED group, but this difference was not significant in ANOVAs performed on the Turn 1 and Leg B signed error scores $(F(1,14)=2.35$ and 2.23 , respectively; both p's $>.05)$. Similar analysis on the slopes and intercepts of the response vs. correct value functions also showed no significant differences $(\mathrm{F}(1,14)=.001,2.24,3.63$, and .06 for Turn 1 slopes, Turn 1 intercepts, Leg B slopes and Leg B intercepts, respectively; all p's > .05).

It is important to note that these Block 2 errors in reaching the intermediate destination were different from Block 1 errors in reaching the final destination, even though both path segments 
were actively controlled. Comparing the Block 1 / Leg C trajectories with Block 2 / Leg B trajectories, the leg and turn path parameters moved in the direction of greater accuracy (increasing slopes and decreasing intercepts), although the changes were not sufficient to produce accurate behavior. Thus, even on the first of two actively-controlled path segments, both groups showed evidence of facilitation in path integration ability. Furthermore, both groups were facilitated to the same extent.

Given that the ON-PATH and ROTATED groups made errors in arriving at the intermediate destination, we may assess the extent to which these intermediate errors affected performance on the final leg. In this regard, the geometry of the stimulus paths is an important consideration. Even though the final destination for the ROTATED and ON-PATH groups is equivalent except for a change in the sign of the turn at Turn 2, this does not mean that similar tendencies to over- or undershoot the correct response values have similar effects upon accuracy in reaching their respective final destinations. If both groups tend to underturn on Turn 1, the stopping location at the intermediate destination will generally be farther away from the final destination for the ONPATH group, and closer to the final destination for the ROTATED group (see Figure 2). Thus, a general pattern of overwalking for the ON-PATH group would tend to cancel errors made in walking to the intermediate destination, to the extent that Legs $\mathrm{B}$ and $\mathrm{C}$ share a common directional component. The same tendency to overwalk in the ROTATED group would tend to increase error rather than diminish it. Conversely, if a tendency to underturn on Turn 1 is repeated on Turn 2, this would tend to increase ON-PATH errors but cancel ROTATED errors.

One way to manage the geometrical considerations is to analyze the responses relative to the correct stopping location at the end of Leg B rather than the participant's actual stopping location. To assess the extent to which the two groups tended to make the same kind of errors on each response path segment (i.e., Turn 1 vs. Turn 2 and Leg B vs. Leg C), we used a least-squares criterion to fit straight lines to each participant's response turns and legs versus the nominally correct response values, for both response path segments. Using ANOVA, we then compared how the slopes and intercepts of these functions changed between the first and second response path segments. This analysis included data from only the ON-PATH and ROTATED groups, and only from Block 2. The comparison of interest is the Segment x Group interaction. If both groups tend to consistently over- or undershoot the nominally correct response values across the two response path segments, there should be no interaction, and the interpretation would be that the observed differences in reaching the final destination are primarily due to the geometry of the "rotated" stimulus configurations. Only the turn intercept analysis showed a significant Segment $\mathrm{x}$ Group interaction $(\mathrm{F}(1,14)=5.55 ; \mathrm{MSe}=3533 ; \underline{p}<.05)$. The lack of interactions in the leg analyses indicate that both groups tended to consistently overwalk relative to the nominally correct response path length, regardless of whether the path was Leg B or Leg C. The consistent overwalking partially explains the greater accuracy for the ON-PATH group in arriving at the final destination-overwalking on Leg B was canceled to some extent by the same overwalking on Leg C. However, the significant Segment $x$ Group interaction in the turn intercept analysis indicates that the group differences cannot entirely be accounted for by differences in path geometry. Specifically, the ON-PATH group executed their turns on Turn 2 in a different manner than they did on Turn 1; in order to arrive accurately at the final destination from their erroneous stopping point at the first target, they needed to execute larger turns than they did on Turn 1, particularly when the nominally correct Turn 2 was relatively shallow. The ON-PATH group made this change and thereby tended to arrive accurately at the origin. By contrast, the ROTATED group would need to make changes both in the execution of Leg $\mathrm{C}$ and in Turn 2 to arrive accurately at their final destination. They made neither change and, as a result, generally missed the final destination.

\section{Discussion}


This study investigated why indirect walking performance is more accurate than performance on triangle completion tasks. At the outset, we considered five hypotheses to explain these performance differences, taking into consideration methodological differences between the two sets of studies as well as fundamental differences between self-directed and experimenterguided movement. Of those five, we conclude that only active control of locomotion clearly facilitated path integration. However, multiple factors are necessary to produce accurate performance in triangle completion tasks.

The basic findings of this study are that participants who saw an off-path marker or a marker adjacent to the origin before performing the triangle completion task showed at best only a small amount of improvement relative to their baseline performance with no visual preview. By contrast, participants in the ON-PATH group showed substantial and reliable improvements due to visual preview of the environment. The information available to the ON-PATH group during the visual preview allowed them to self-direct motion through the first turn and second leg of the path before returning to the origin. Although they made errors in reaching intermediate destinations, they corrected for these errors and arrived accurately back at the origin, despite the fact that they received no further positional information while executing the response. The ROTATED group, who differed from the ON-PATH group in having a final destination other than the origin, made similar errors in reaching intermediate destinations, but performed quite poorly at reaching the final destination. Similar erroneous response tendencies in reaching the intermediate destination would have different consequences for the ROTATED and ON-PATH groups, in terms of the trajectories required to arrive accurately at the final destination. At least some of the lesser accuracy of the ROTATED group appears attributable to these differences in the required final trajectory. Note, moreover, that despite the ROTATED group's greater error in the final stopping points, the ONPATH and ROTATED terminal locations were each tightly clustered.

We consider these results in terms of the five hypotheses mentioned in the introduction. With regard to Hypothesis (1), the OFF-PATH and ORIGIN groups responses showed that there was no general benefit of having available a memory of the spatial structure of the environment. The lack of clear improvement in these groups also discounts Hypotheses (2) and (3) concerning the possible benefit of having nearby remembered landmarks and of having a salient, well-localized marker at the destination. ${ }^{3}$ The data clearly do not support Hypothesis (5), which proposed that path integration may be improved when action is directed to a non-origin destination. The ROTATED group performed inaccurately in attempting to reach each of two non-origin targets, and the ON-PATH group performed well in reaching the final destination even though it was the origin. With regard to the possible benefit of increasing the proportion of actively-controlled path segments (Hypothesis 4), active control of locomotion clearly facilitates performance, even though it does not necessarily produce accurate performance. Both of the "active" groups (ON-PATH and ROTATED) were relatively inaccurate at reaching an intermediate destination, despite the fact that they attempted to walk to that location under their own control, and only the ON-PATH group arrived accurately at the final destination after visual previews. However, when visual previews allowed the participants to actively control a higher proportion of their trajectories, the response turn and response path length errors (relative to the nominally correct values) were much reduced for both groups. Greater active control was also associated with increased convergence of final trajectories upon a common location.

A surprising finding is that after walking inaccurately to the intermediate destination, the ON-PATH group then adjusted their trajectories on the second response path segment (consciously or unconsciously) in such a way as to improve their final accuracy, whereas the ROTATED group did not. That is, although both groups benefited from active control, accuracy in reaching the final destination was increased when active control was used to direct action toward the origin of locomotion. The question that remains unanswered is: how does the origin come to achieve this 
apparent "special status" when there is a substantial amount of active control? As we have seen, there are no striking advantages associated with simply returning to the origin-indeed, the typical triangle completion task involves returning to the origin and yet performance is generally poor. The performance of the ON-PATH group suggests that updating of the final goal was performed at the expense of updating the intermediate destination. In the case of the ROTATED group, this final goal apparently did not provide a strong stimulus to updating. Several possible reasons for this are the following:

(1) Errors in perceptual localization of the destination: The final destinations for the ROTATED group were not coincident with the participant's initial viewpoint, and thus were relatively imprecisely localized. This relative imprecision could have been enhanced as the experiment proceeded because these locations changed from trial to trial. It may be that when participants always return to the same location, as did the ON-PATH group, a benefit develops as the number of trials increases, such that the accumulating experience of attempting to return to the same location in space helps to consolidate the representation of that location within the context of the remembered environment. This consolidation could improve path integration when action is directed to that location, regardless of whether or not that location is the origin. The ON-PATH group returned to a common destination (the origin) on all trials, whereas the ROTATED group did not, instead returning to one of six possible locations that varied from trial to trial. This difference suggests that had the ROTATED group always attempted to walk to the same non-origin final destination, their performance would improve.

(2) Errors in path integration: Although the ROTATED group's path integration ability was enhanced in Block 2, the benefits were still insufficient to produce accurate behavior. The ROTATED group made similar kinds of errors on both the first and second actively-controlled path segments in Block 2. As mentioned above, relatively small and consistent errors in path integration across path segments tended to magnify systematic errors in reaching the final destination, due to the geometry of the ROTATED stimulus paths. In addition, given the systematic errors in reaching the intermediate destination, the amount of compensation required to produce accurate performance was much greater for the ROTATED group: whereas the ON-PATH group only needed to overturn on Turn 2, the ROTATED group had to both underturn on Turn 2 and produce Leg $\mathrm{C}$ lengths that were substantially shorter than the ideal Leg C. It may be that participants can compensate for systematic errors in path integration if only a small amount of compensation is required. In any case, if systematic error in path integration in indeed the primary cause of the ROTATED group's poor performance in reaching the final destination, it is significant that these systematic errors nevertheless preserved the convergence of the walking trajectories.

(3) Errors in updating the final destination: It may be that updating the origin's location is obligatory, in the sense that doing so consumes processing resources even if returning to the origin is not an explicit goal. If this is true, the advantage provided by active locomotion might be nullified by performance decrements stemming from the requirement of updating an additional location: whereas the ON-PATH group must only update the location of the intermediate destination and the origin, the ROTATED group must update the origin plus the intermediate and final destinations. The additional processing load could introduce systematic errors in the represented location of the final target. Walking directed toward this represented location, however, could be carried out accurately, resulting in a convergence of walking trajectories upon a location displaced from the target destination. Clearly, specifying the role of the origin lies at the heart of answering several important questions in human path integration.

The complexities of the ROTATED group's performance notwithstanding, this experiment yielded several unambiguous results. In the context of triangle completion (i.e., returning to the origin), providing vision of a structured environment before the task begins is not, by itself, sufficient to support good performance. By contrast, vision that provides information for active 
control of locomotion can substantially enhance path integration ability. This enhancement is not obligatory, however, and a variety of factors can introduce systematic errors. Foremost among these factors is the requirement of walking to multiple targets; this can cause one target to be updated at the expense of another. Systematic errors are also associated with attempting to walk to a final destination that is displaced from the origin, although the reasons for this association are not yet known. Exploring the link between active control and how action is directed toward particular locations in space promises to yield important insights not only into human path integration but also into the coupling between perception and action. 


\section{References}

Berthoz, A., Amorim, M.-A., Glasauer, S., Grasso, R., Takei, Y., \& Viaud-Delmon, I. (1999). Dissociation between distance and direction during locomotor navigation. In R. G. Golledge (Ed.), Wayfinding behavior: Cognitive mapping and other spatial processes (pp. 328348). Baltimore: Johns Hopkins University Press.

Corlett, J. (1992). The role of vision in the planning and guidance of locomotion through the environment. In L. Proteau \& D. Elliott (Eds.), Vision and motor control (pp. 375-397): Elsevier Science.

Elliott, D. (1987). The influence of walking speed and prior practice on locomotor distance estimation. Journal of Motor Behavior, 19, 476-485.

Etienne, A. S., Maurer, R., Berlie, J., Reverdin, B., Rowe, T., Georgakopoulos, J., \& Séguinot, V. (1998). Navigation through vector addition. Nature, 396, 161-164.

Festinger, L., Ono, H., \& Burnham, C. A. (1967). Efference and the conscious experience of perception. Journal of Experimental Psychology, 74, 1-36.

Fujita, N., Klatzky, R. L., Loomis, J. M., \& Golledge, R. G. (1993). The encoding-error model of pathway completion without vision. Geographical Analysis, 25, 295-314.

Fukusima, S. S., Loomis, J. M., \& Da Silva, J. A. (1997). Visual perception of egocentric distance as assessed by triangulation. Journal of Experimental Psychology: Human Perception and Performance, 23, 86-100.

Gallistel, C. R. (1990). The organization of learning. Cambridge, MA: MIT Press.

Gladwin, T. (1990). East is a big bird: Navigation and logic on Puluwat Atoll. Cambridge, MA: Harvard University Press.

von Helmholtz, H. E. F. (1962). Treatise on physiological optics (J. P. C. Southall, Trans.). New York: Dover.

Hutchins, E. (1995). Cognition in the wild. Cambridge, MA: MIT Press.

Kelso, J. A. (1977). Motor control mechanisms underlying human movement reproduction. Journal of Experimental Psychology: Human Perception \& Performance, 3, 529543.

Klatzky, R. L., Beall, A. C., Loomis, J. M., Golledge, R. G., \& Philbeck, J. W. (1999). Human navigation ability: Tests of the encoding-error model of path integration. Spatial Cognition and Computation, 1, 31-65.

Klatzky, R. L., Loomis, J. M., Golledge, R. G., Cicinelli, J. G., Doherty, S., \& Pellegrino, J. W. (1990). Acquisition of route and survey knowledge in the absence of vision. Journal of Motor Behavior, 22, 19-43.

Loomis, J. M., Da Silva, J. A., Fujita, N., \& Fukusima, S. S. (1992). Visual space perception and visually directed action. Journal of Experimental Psychology: Human Perception \& Performance, 18, 906-921. 
Loomis, J. M., Klatzky, R. L., Golledge, R. G., Cicinelli, J. G., Pellegrino, J. W., \& Fry, P. A. (1993). Nonvisual navigation by blind and sighted: Assessment of path integration ability. Journal of Experimental Psychology: General, 122, 73-91.

Loomis, J. M., Klatzky, R. L., Golledge, R. G., \& Philbeck, J. W. (1999). Human navigation by path integration. In R. G. Golledge (Ed.), Wayfinding behavior: Cognitive mapping and other spatial processes (pp. 125-151). Baltimore, MD: Johns Hopkins Press.

Loomis, J. M., Klatzky, R. L., Philbeck, J. W., \& Golledge, R. (1998). Assessing auditory distance perception using perceptually directed action. Perception \& Psychophysics, 60, 966-980.

Pepper, R. L., \& Herman, L. M. (1970). Decay and interference effects in the short-term retention of a discrete motor act. Journal of Experimental Psychology, 83, 1-18.

Philbeck, J. W., Behrmann, M., Black, S. E., \& Ebert, P. (in press). Intact spatial updating during locomotion after right posterior parietal lesions. Neuropsychologia.

Philbeck, J. W., \& Loomis, J. M. (1997). Comparison of two indicators of perceived egocentric distance under full-cue and reduced-cue conditions. Journal of Experimental Psychology: Human Perception \& Performance, 23, 72-85.

Philbeck, J. W., Loomis, J. M., \& Beall, A. C. (1997). Visually perceived location is an invariant in the control of action. Perception \& Psychophysics, 59, 601-612.

Rieser, J. J. (1999). Dynamic spatial orientation and the coupling of representation and action. In R. G. Golledge (Ed.), Wayfinding behavior: Cognitive mapping and other spatial processes (pp. 168-190). Baltimore, MD: Johns Hopkins Press.

Rieser, J. J., Ashmead, D. H., Talor, C. R., \& Youngquist, G. A. (1990). Visual perception and the guidance of locomotion without vision to previously seen targets. Perception, $\underline{19}, 675-689$.

Rieser, J. J., \& Frymire, M. (1995). Locomotion with vision is coupled with knowledge of real and imagined surroundings. Paper presented at the Psychonomics Society, Los Angeles, CA.

Rieser, J. J., Frymire, M., \& Berry, D. (1997). Geometrical constraints on imagery and action when walking without vision. Paper presented at the Psychonomic Society, Philadelphia, PA.

Sadalla, E. K., \& Montello, D. R. (1989). Remembering changes in direction. Environment and Behavior, 19, 614-635.

Sholl, M. J. (1989). The relation between horizontality and rod-and-frame and vestibular navigation performance. Journal of Experimental Psychology: Learning, Memory, and Cognition, $\underline{15}, 110-125$.

Srinivasan, M., Zhang, S., \& Bidwell, N. (1997). Visually mediated odometry in honeybees. Journal of Experimental Biology, 200, 2513-2522.

Steenhuis, R. E., \& Goodale, M. A. (1988). The effects of time and distance on accuracy of target-directed locomotion: Does an accurate short-term memory for spatial location exist? Journal of Motor Behavior, 20, 399-415. 
Summers, J. J., Levey, A. J., \& Wrigley, W. J. (1981). The role of planning and efference in the recall of location and distance cues in short-term motor memory. Journal of Motor Behavior, 13, 65-76.

Thomson, J. A. (1983). Is continuous visual monitoring necessary in visually guided locomotion? Journal of Experimental Psychology: Human Perception \& Performance, 9, 427-443.

\author{
Author Notes
}

This work was supported by NIH grant F32 MH11791 and JSMF grant 97-17 to J.W.P. and NIH grant MH 54246 to M.B. We thank Anthony Cate, Connie Deighan, Catherine Ewell, and Thomas McKeeff for assistance in conducting the experiment and Anthony Wingen for assistance in scheduling. We also thank Ken Cheng, Digby Elliott and an anonymous reviewer for helpful comments on earlier versions of this paper.

Correspondence and requests for reprints should be addressed to John W. Philbeck, Department of Psychology, Carnegie Mellon University, Pittsburgh, PA 15213 (e-mail: philbeck@andrew.cmu.edu).

\title{
Footnotes
}

${ }^{1}$ If the participants updated with reference to a general representation of the wall facing them during the visual preview, neither workspace would provide an advantage because the location of the starting position relative to the wall was the same in both spaces. The possibility remains, however, that the participants could have updated relative to some specific environmental feature on the wall. Although we cannot rule out this possibility, we think it unlikely for several reasons. From an information processing standpoint, updating relative to an environmental feature at these distances would be a poor strategy. Any individual feature on the far wall was too near to provide a reliable azimuthal reference as the participant walked; on the other hand, these features lay well beyond the experimenter-defined targets and thus would provide weaker stimuli to imagined motion parallax than the nominal targets. From an empirical standpoint, as we will see, the performance of the ON-PATH and ROTATED groups in walking to the intermediate destination was statistically indistinguishable, even though 6 participants in the ON-PATH group viewed the smaller workspace before walking compared with only 3 in the ROTATED group.

${ }^{2}$ Due to an error in calculating the nominal coordinates of the "rotated" stimulus destinations, one of the stimulus destinations was displaced from its geometrically ideal location by about $1 \mathrm{~m}$. This affected 2 of the 6 experimental paths, and resulted in only minor changes in the ideal response turn and path lengths on those paths relative to the ideal values for the other groups.

${ }^{3}$ Although these results may appear to be in conflict with those of Rieser and his colleagues (Rieser \& Frymire, 1995; Rieser et al., 1997), they do not rule out the possibility that under other circumstances there is a benefit associated with calling to memory a spatial structure or paying attention to particular remembered landmarks. Influences of remembered spatial structure on path integration may be more apparent when the stimulus paths are more complex or when the participants are trained or instructed differently. Another possibility is that a reduction in the complexity of the surrounding spatial structure (e.g., by presenting a small number of glowing stimuli in the dark) may heighten the influence of very specific markers. 
Table 1

Source Table for Analysis of Variance (ANOVA) of Response Parameters

\begin{tabular}{crrc}
\hline Response Parameters & MSe & F-ratio & p-value \\
\hline Leg C Slope & & & \\
Group $(3,28)$ & 0.08 & 1.74 & $>.05$ \\
Block $(1,28)$ & 0.20 & 11.20 & 0.0023 \\
Block * Group $(3,28)$ & 0.11 & 6.19 & 0.0023 \\
& & & \\
Leg C Intercept & & & \\
Group $(3,28)$ & 5.05 & 8.69 & 0.0003 \\
Block $(1,28)$ & 0.94 & 2.48 & $>.05$ \\
Block * Group $(3,28)$ & 1.63 & 4.28 & 0.0132 \\
Turn 2 Slope & & & \\
Group $(3,28)$ & 0.06 & 1.00 & $>.05$ \\
Block $(1,28)$ & 0.13 & 6.66 & 0.0154 \\
Block * Group $(3,28)$ & 0.09 & 4.65 & 0.0093 \\
Turn 2 Intercept & & & \\
Group (3, 28) & 1359 & 1.28 & $>.05$ \\
Block $(1,28)$ & 4218 & 8.35 & 0.0074 \\
Block * Group $(3,28)$ & 863 & 1.71 & $>.05$
\end{tabular}




\section{Figure Captions}

Figure 1. Stimulus path configurations and landmark locations. (a) Sample stimulus trajectories and correct response trajectories in Block 2 (Visual Preview) for the four experimental groups. (b) Experimental stimulus paths; black dots mark possible drop-off points at the end of the second leg of the triangle. The first leg was of constant length. (c) "Dummy" stimulus paths; symbols are as for the experimental paths. (d) The four possible locations of "off-path" markers for the OFFPATH group in Block 2 (outline crosses). The possible drop-off points are also shown, along with one experimental stimulus path. (e) The six possible locations for final destination markers for the ROTATED group in Block 2 (outline crosses). The possible drop-off points are shown, along with one experimental stimulus path (solid line). An ideal response path back to the origin is marked with a thin dashed line, and the ideal response path to its corresponding "rotated" destination (reflected about the second path segment) is marked with a heavy dashed line. The small cross on the left of panel (e) shows the geometrically correct "rotated" destination of one of the experimental paths; the outlined cross just above it shows the location actually used in the experiment.

Figure 2. Centroids of the final stopping points of the response trajectories, computed across the eight participants in each group. Data for the four experimental groups appears in successive rows, with Block 1 (No Visual Preview) performance shown in the left-most column and Block 2 (Visual Preview) performance shown in the middle and right-most columns. In the Block 2 data for the ON-PATH and ROTATED groups, small black dots show the centroids of the locations at which the participants stopped to indicate the location of the intermediate destination at the end of Leg B. Dotted lines connect the centroids of the Leg B endpoints and the centroids of the final stopping locations. If performance were correct, the dotted lines would emanate from the large dots designating the experimenter-defined endpoints of the various Leg B's and converge upon the origin (or upon the outlined crosses for the ROTATED group, Block 2). For clarity, the Experimental and Dummy Trials are shown separately for the ON-PATH and ROTATED groups in Block 2.

Figure 3. Average parameters (across participants) of the best-fitting straight lines through the Leg $\mathrm{C}$ and Turn 2 data, for Block 1 (No Visual Preview) and Block 2 (Visual Preview). (a) and (b) Response Turn 2 slopes and intercepts. (c) and (d) Response Leg C slopes and intercepts. The parameters were computed for each participant by plotting the response values as a function of the correct values and finding the parameters of the best-fitting straight line through the data by the least-squares criterion. For the ON-PATH and ROTATED groups, the correct responses in Block 2 were defined relative to the actual stopping point at the end of Leg B. Error bars show +/- one standard error of the mean. 
(a)

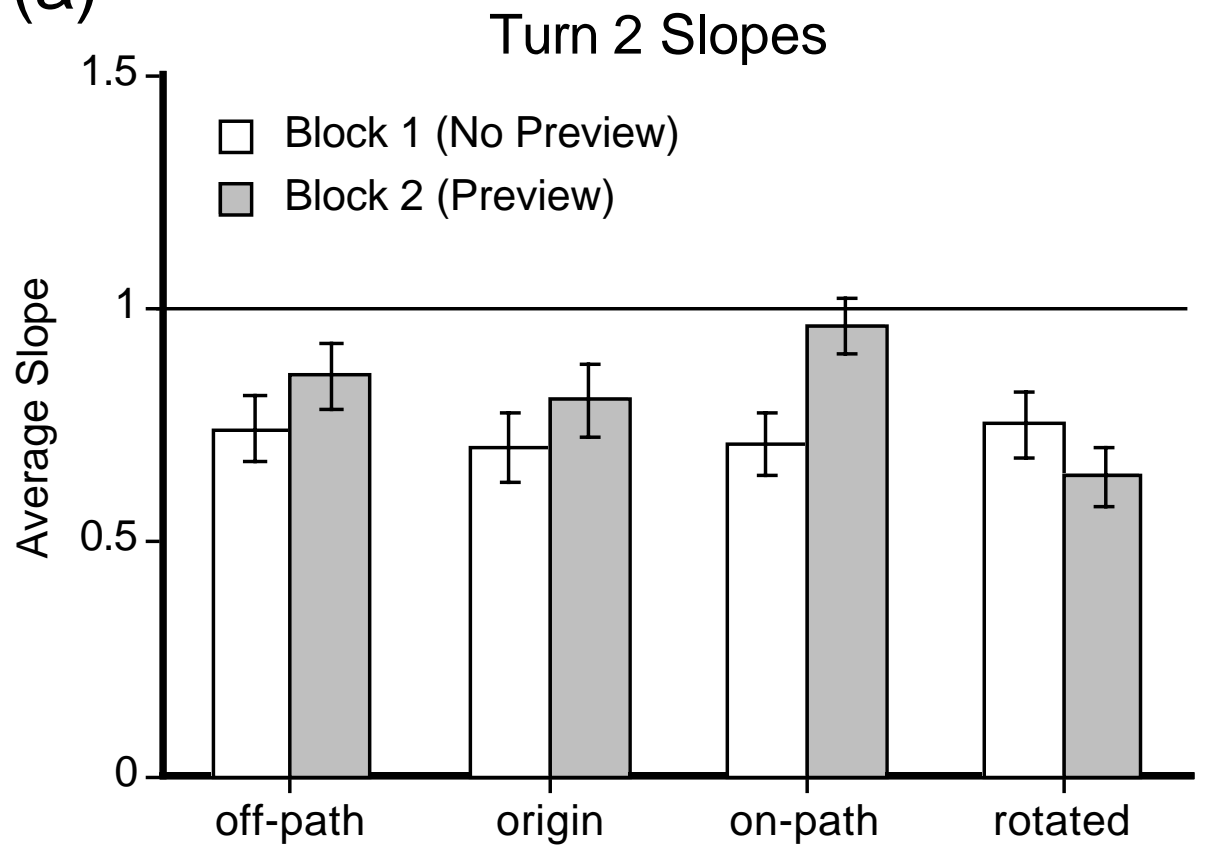

(c)

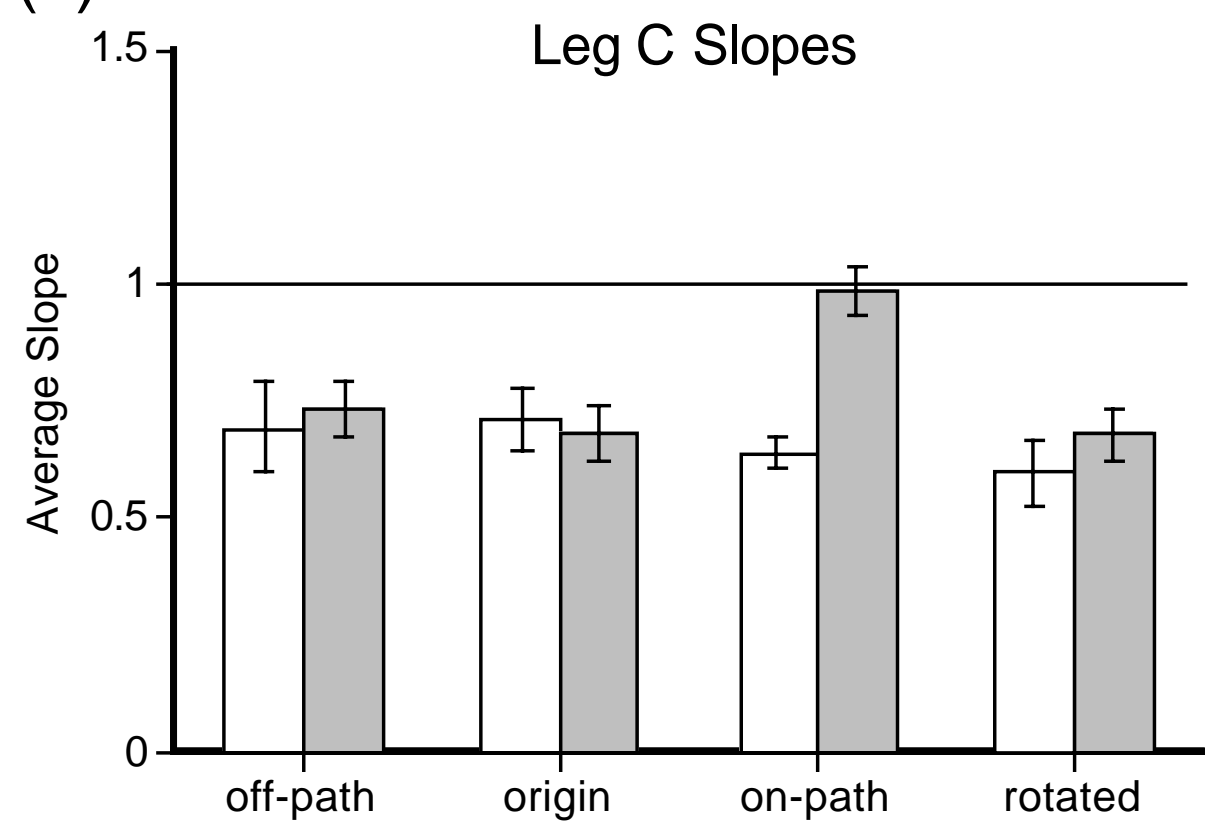

(b)

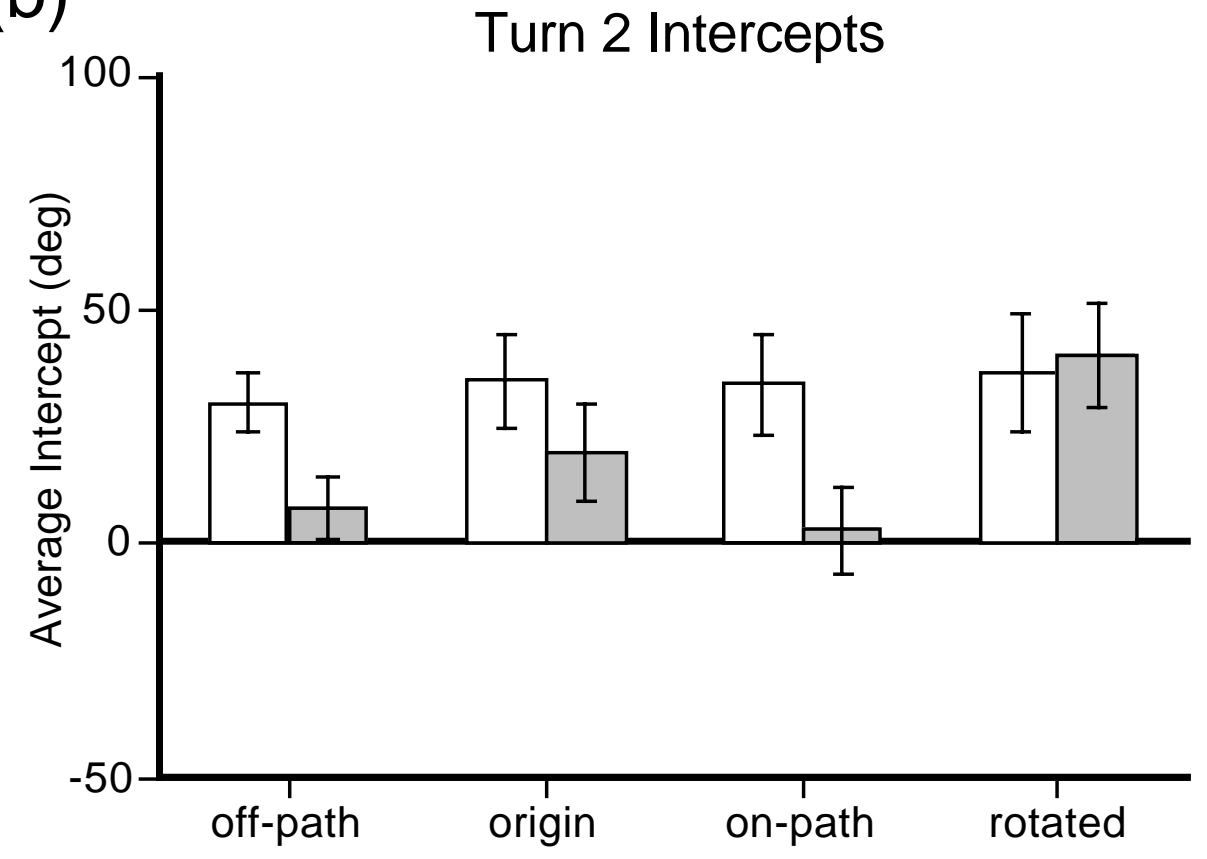

(d)

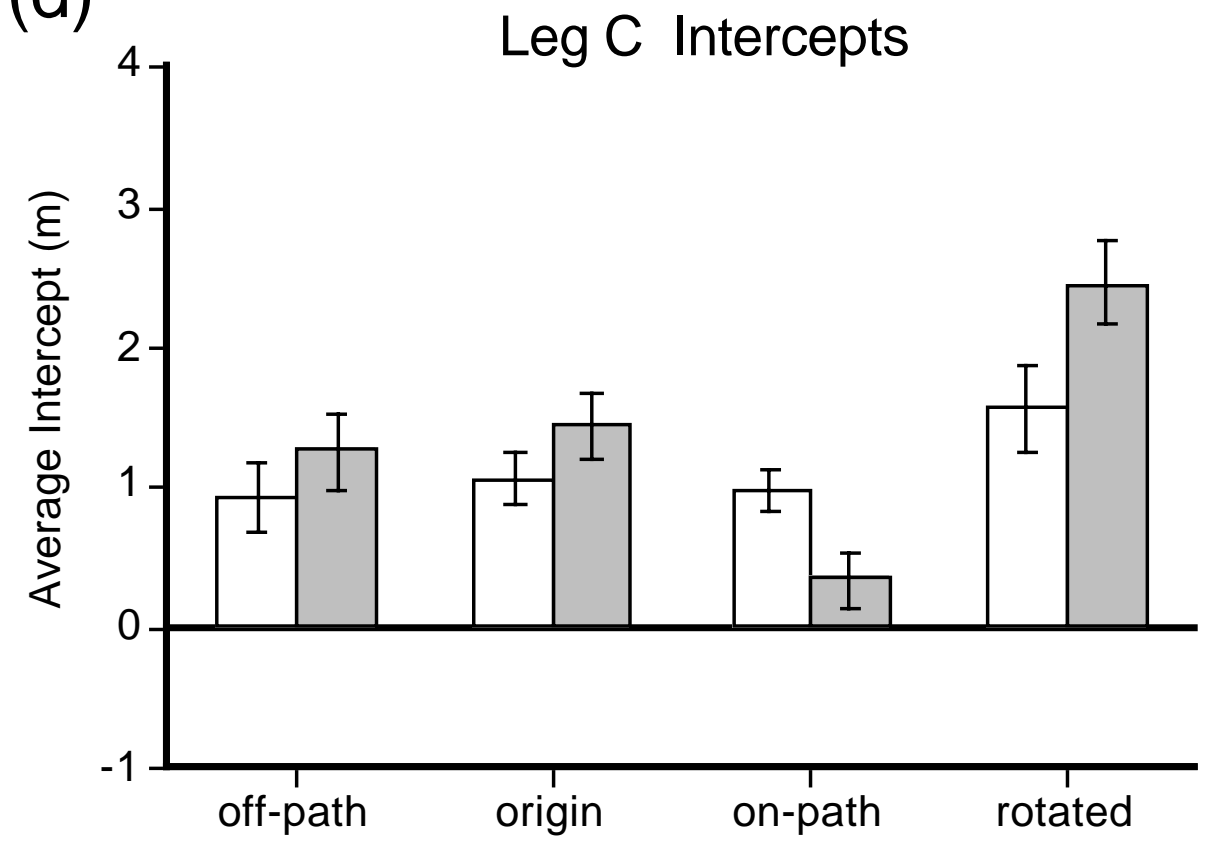

Figure 3 


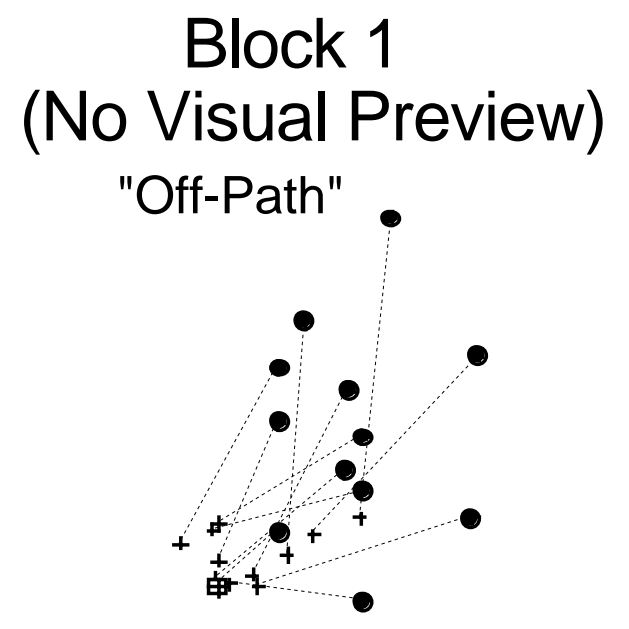

"Origin"

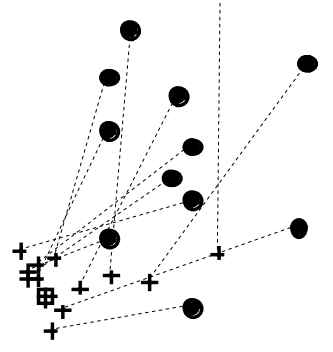

"On-Path" •
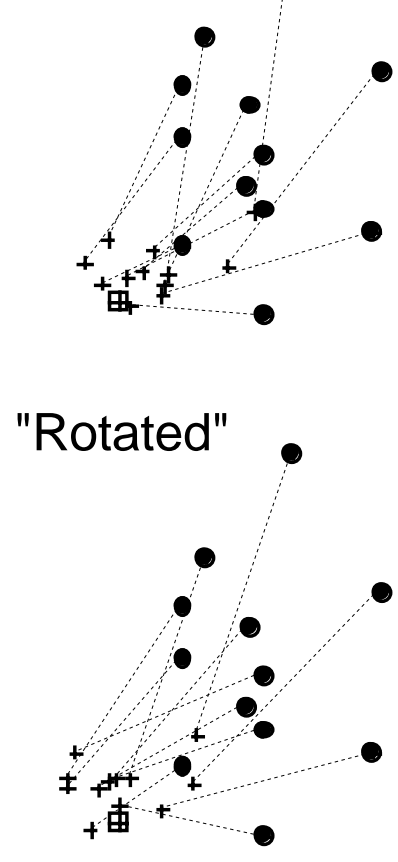

Block 2

(Visual Preview)

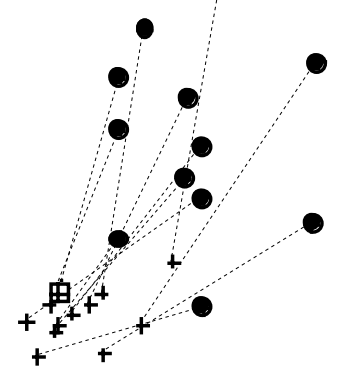

田 Origin

- End of Leg B

(Experimenter-Defined)

- End of Leg B

(Participant-Generated)

$+\quad \begin{aligned} & \text { Centroids of Final } \\ & \text { Stopping Points }\end{aligned}$

$\nLeftarrow$ "Rotated" Destinations
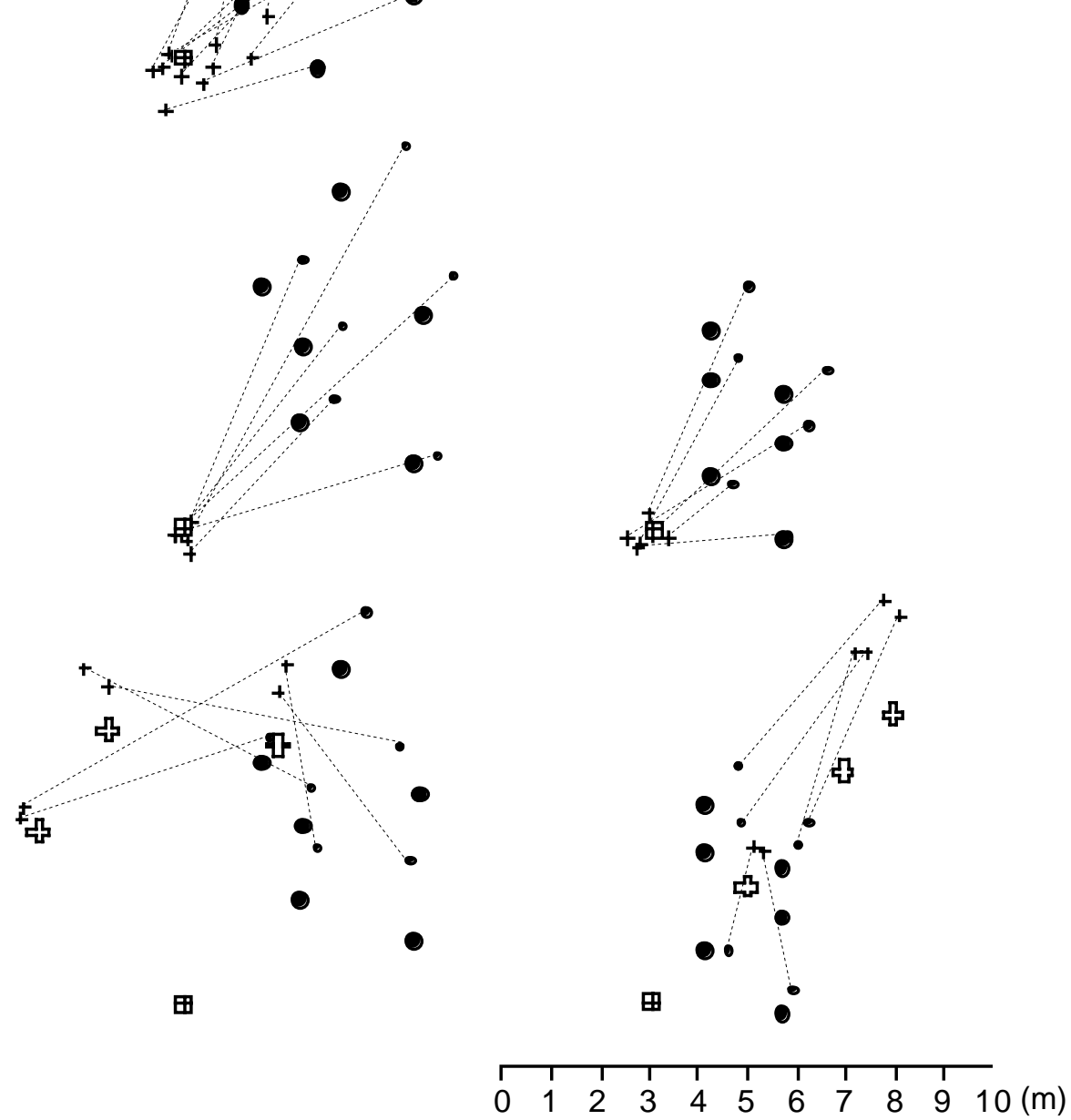

Figure 2 
(a)

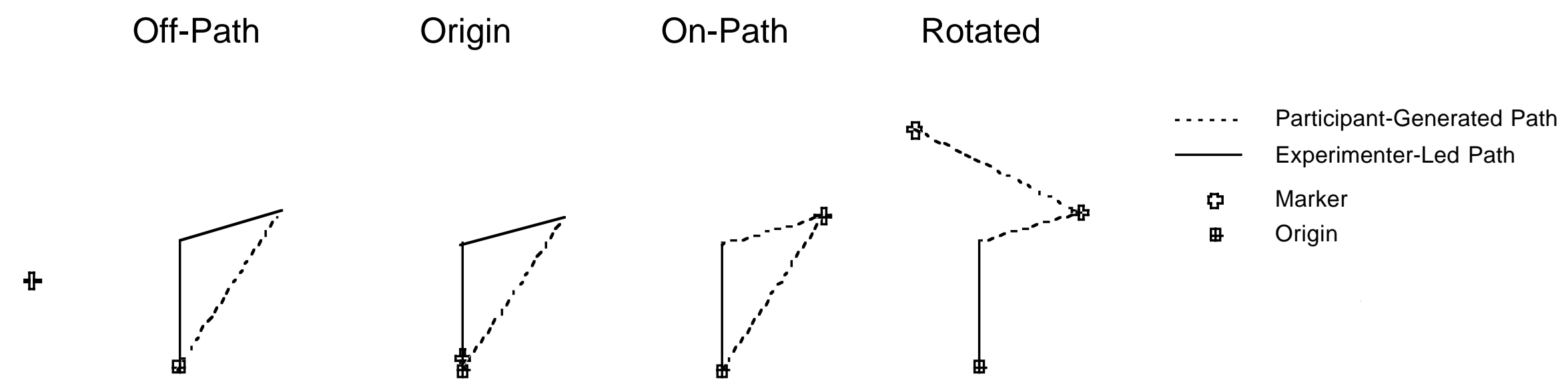

(b)

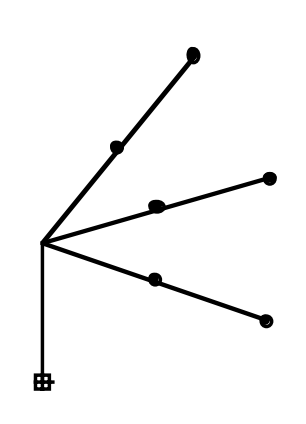

origin (c)

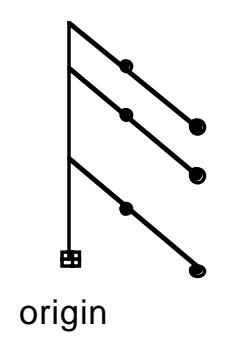

(d)

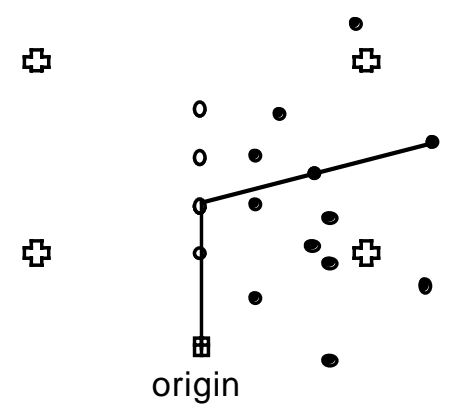

(e)

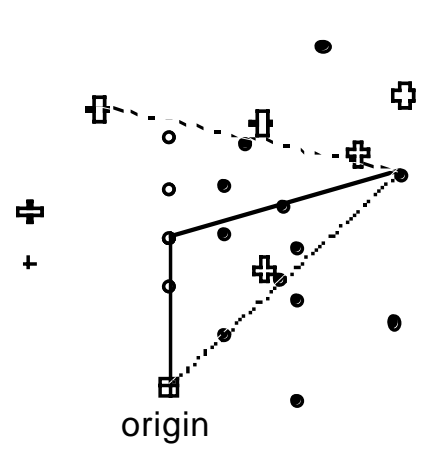

$\left.\begin{array}{cccccccccccc}-4-3-2 & -1 & 1 & 1 & 1 & 1 & 1 & 1 & 1 & 1\end{array}\right](\mathrm{m})$

Figure 1 